\title{
Minimal Surfaces, Screw Dislocations and Twist Grain Boundaries
}

\author{
Randall D. Kamien ${ }^{\dagger}$ and T.C. Lubensky ${ }^{\ddagger}$ \\ Department of Physics and Astronomy, University of Pennsylvania, Philadelphia, PA 19104, USA \\ Large twist-angle grain boundaries in layered structures are often described by \\ Scherk's first surface whereas small twist-angle grain boundaries are usually described \\ in terms of an array of screw dislocations. We show that there is no essential distinc- \\ tion between these two descriptions and that, in particular, their comparative energetics \\ depends crucially on the core structure of their screw-dislocation topological defects.
}

27 August 1998; revised 2 November 1998

\footnotetext{
$\dagger$ email: kamien@physics.upenn.edu

$\ddagger$ email: tom@lubensky.physics.upenn.edu
} 
Contrary to their name, minimal surfaces are widely observed. It has been proposed [1] that lamellae in diblock copolymer systems form minimal surfaces so as to minimize the interfacial area between blocks. Other systems, such as cubic phases [2] and the smectic-Q phase [3] are also described in this way. These minimal surfaces contain grain boundaries, which rotate the layer normals. On the other hand, a twist-grain-boundary (TGB) in a smectic-A liquid crystal phase is described in terms of a lattice of screw dislocations [4]. We will show that these two different descriptions lead to nearly identical geometrical surfaces even though the philosophy behind their construction is quite different.

Minimal surfaces are simply surfaces of zero mean curvature. Because of this, they play an important role in the physics of complex fluids: their curvature energy always vanishes. Though they may have periodically repeated unit cells or, like Scherk's first surface [5], provide a continuous transition between parallel planes at different angles, they do not have an inherent length scale - a uniform dilation of a minimal surface is still minimal. One of the simplest minimal surfaces is the helicoid: a surface with height function $h(x, y)=h_{0}+[b /(2 \pi)] \tan ^{-1}(y / x)$. This surface is smooth and continuous, and there are no constraints on the parameter $b$ giving the pitch of the helicoid. A screw dislocation in a smectic-A, however, is a topological defect in a lamellar structure with a specified periodic spacing $d$. It has a height function identical to that of a helicoid, though its pitch must be an integer multiple of the layer spacing. As we discuss below, this constraint is most easily implemented in an Eulerian picture in which the single-valued phase of the mass-density wave is spatially dependent. Moreover, a true dislocation has a singular core because the layer spacing and thus the strain diverges there. This costs energy precisely because there is a preferred layer spacing. Thus the existence of a well-defined periodic reference state distinguishes screw dislocations from helicoids. In this letter, we will explore the consequences of this distinction.

We start by considering Scherk's first surface. The height function $h(x, y)$ is given implicitly via [5]:

$$
x \sin \alpha=\ln \left[\frac{\cos \left[\cos \left(\frac{\alpha}{2}\right) h(x, y)+\sin (\alpha / 2) y\right]}{\cos \left[\cos \left(\frac{\alpha}{2}\right) h(x, y)-\sin (\alpha / 2) y\right]}\right]=\ln \left[\frac{\cos \left(\mathbf{N}_{1} \cdot \mathbf{t}\right)}{\cos \left(\mathbf{N}_{2} \cdot \mathbf{t}\right)}\right]
$$

where $\mathbf{t} \equiv(x, y, h(x, y))$ and $\mathbf{N}_{1,2}=(0, \pm \sin (\alpha / 2), \cos (\alpha / 2)$ are unit vectors. By using the rightmost expression in (1), it is easy to see that as $x \rightarrow-\infty$ (for $0<\alpha<\frac{\pi}{2}$ ) the surface is the solution of $\cos \left(\mathbf{N}_{1} \cdot \mathbf{t}\right)=0$, i.e., Scherk's surface is a family of parallel planes 
with layer normal $\mathbf{N}_{1}$ and spacing $d=\pi$. Similarly, as $x \rightarrow \infty$ Scherk's surface becomes a family of parallel layers with normal $\mathbf{N}_{2}$ and spacing $d=\pi$.

Note that as $\alpha \rightarrow 0$, (西) becomes

$$
x \sin \alpha \approx \ln [1-2 y \sin (\alpha / 2) \tan h] \approx-y \sin \alpha \tan h
$$

so that

$$
h(x, y)=\frac{\pi}{2}+\tan ^{-1}\left(\frac{y}{x}\right)
$$

which is a single helicoid with pitch $p=2 \pi=2 d$ located at the origin. Since planes at infinity are separated by $\pi$, this can be interpreted as a +2 screw dislocation. Note, however, that the normal distance between the planes diverges along the $\hat{z}$-axis, a fact that will prove to be essential in our discussion of energetics. In biphasic materials, such as diblock copolymers, these layers should be alternately identified as A-blocks and B-blocks. This would make the layer spacing at infinity $\tilde{d}=2 d=2 \pi$ and thus the Burgers vector $b=2 \pi$ would be a strength +1 dislocation [1]. However, in the following we will take each layer to be identical. It will only be convention as to the strength of the dislocation - the actual length of the Burgers vector is the appropriate invariant.

For finite $\alpha$, the intersection of the Scherk's surface with the plane $x=0$ consists of two sets of equally spaced lines: one parallel to the $\hat{z}$-axis at positions $y_{k}=k l_{d}$ for integer $k$, with separation $l_{d}=\pi / \sin (\alpha / 2)=d / \sin (\alpha / 2)$ and a second set parallel to $\hat{y}$-axis at the heights $h_{k}=k d^{\prime}$ with separation $d^{\prime}=\pi / \cos (\alpha / 2)=d / \cos (\alpha / 2)$. Around the first set of lines, Scherk's surface is a local helicoid with height function $h(x, y)=$ $-[1 / \cos (\alpha / 2)] \tan ^{-1}[x \cos (\alpha / 2) / \delta y]$ for small $\delta y=y-y_{k}$. Thus these helicoids have pitch $p_{z}=2 d^{\prime}$ equal to twice the separation between the planes parallel to $\hat{y}$ at $x=$ 0. Similarly, around the second set of lines there are helicoids: for small $\delta h=h-h_{k}$, $y(x, h)=-[1 / \sin (\alpha / 2)] \tan ^{-1}[x \sin (\alpha / 2) / \delta h]$. Thus they have pitch $p_{y}=2 l_{d}$ equal to twice the separation between planes parallel to $\hat{z}$ at $x=0$. Note that neither $p_{z}$ or $p_{y}$ are integer multiples of the layer spacing at infinity $d$. The two sets of helicoids can be interchanged through the "dual" transformation $h(x, y) \leftrightarrow y$ and $\alpha \rightarrow \pi-\alpha$ which leaves Scherk's surface invariant and interchanges $d^{\prime}$ and $l_{d}$. In the following we shall show that if the (non-chiral) ground state of the system is lamellar, with a preferred layer spacing, then energetics will break the geometric degeneracy of the dual mapping by converting one 
set of helicoids into true topological screw dislocations with cores. Finally, for comparison with the smectic structures we will consider, we calculate the derivatives of $h(x, y)$ :

$$
\begin{aligned}
\partial_{x} h(x, y) & =-\sin (\alpha / 2) \frac{\sin (2 \theta)}{\cosh (2 \gamma)-\cos (2 \theta)} \\
\partial_{y} h(x, y) & =\tan (\alpha / 2) \frac{\sinh (2 \gamma)}{\cosh (2 \gamma)-\cos (2 \theta)}
\end{aligned}
$$

where $\theta \equiv y \sin (\alpha / 2)$ and $\gamma \equiv \frac{1}{2} x \sin \alpha$. From these derivatives and boundary conditions Scherk's surface can be reconstructed.

We will now consider a surface constructed via a linear superposition of screw dislocations (LSD) in a smectic-A phase, parallel to $\hat{z}$ with separation $l_{d}$ along the $\hat{y}$-axis. Recall that a smectic is described in terms of a complex scalar mass-density wave

$$
\psi=|\psi| e^{i q[z-\bar{u}(x, y, z)]}
$$

where $q=2 \pi / d$ and $d$ is the layer spacing. While the surface $h(x, y)$ is a true height function expressed in Lagrangian coördinates, smectics are described in terms of the phase variable $\bar{u}$, which is Eulerian. To compare with Scherk's surface we shall take $d=\pi$ here and in the following. Because $\psi$ must be single valued, the Burgers vector $b$ must be an integer multiple of the layer spacing. The (Lagrangian) surfaces of constant phase $\bar{h}(x, y)$ are defined implicitly through

$$
\bar{h}(x, y)-\bar{u}(x, y, \bar{h}(x, y))=k d
$$

where $k$ is an integer. Thus $\partial_{x} \bar{h}=\partial_{x} \bar{u} /\left(1-\partial_{z} \bar{u}\right)$ and similarly for $\partial_{y} \bar{h}$. For low-angle grain-boundaries, $\partial_{z} \bar{u} \approx 0$ and the derivatives of $\bar{h}$ and $\bar{u}$ are equal. This will not be the case for large angle grain-boundaries. With this in mind, we will add together the effect of a plane of equally spaced screw dislocations with spacing $l_{d}$.

A single screw dislocation is described by $\bar{u}_{1}=[b /(2 \pi)] \tan ^{-1}(y / x)$ with $b=k d$ :

$$
\left[\partial_{x} \bar{u}_{1}, \partial_{y} \bar{u}_{1}, \partial_{z} \bar{u}_{1}\right]=\frac{b}{2 \pi}\left[\frac{-y}{x^{2}+y^{2}}, \frac{x}{x^{2}+y^{2}}, 0\right]
$$

An array of screw dislocations gives:

$$
\begin{aligned}
& \partial_{x} \bar{u}_{\text {array }}=-\frac{b}{2 \pi} \sum_{n=-\infty}^{\infty} \frac{y-n l_{d}}{x^{2}+\left(y-n l_{d}\right)^{2}} \\
& \partial_{y} \bar{u}_{\text {array }}=\frac{b}{2 \pi} \sum_{n=-\infty}^{\infty} \frac{x}{x^{2}+\left(y-n l_{d}\right)^{2}} .
\end{aligned}
$$


These sums can be performed explicitly via the Poisson summation formula:

$$
\begin{aligned}
\partial_{x} \bar{u}_{\text {array }} & =-\frac{b}{2 l_{d}} \frac{\sin \left(2 \pi y / l_{d}\right)}{\cosh \left(2 \pi x / l_{d}\right)-\cos \left(2 \pi y / l_{d}\right)} \\
\partial_{y} \bar{u}_{\text {array }} & =\frac{b}{2 l_{d}} \frac{\sinh \left(2 \pi x / l_{d}\right)}{\cosh \left(2 \pi x / l_{d}\right)-\cos \left(2 \pi y / l_{d}\right)} .
\end{aligned}
$$

Note that the superposition of (7) gives $\partial_{z} \bar{u}_{\text {array }}=0$, and thus $\bar{u}_{\text {array }}$ is independent of $z$. However, $\bar{u}_{\text {array }}$ is the displacement due only to the screw dislocations - the smectic will relax via a smooth displacement field $\bar{u}_{\text {smooth }}(x, y, z)$. To find $\bar{u}_{\text {smooth }}$ we consider the boundary condition on the smectic: the strain must vanish at $x= \pm \infty$. Writing $\bar{u}=\bar{u}_{\text {array }}+\bar{u}_{\text {smooth }}$, the rotationally invariant strain is $u_{z z}=\partial_{z} \bar{u}-\frac{1}{2}(\nabla \bar{u})^{2}$ and the boundary condition becomes:

$$
2 \partial_{z} \bar{u}-\left(\partial_{z} \bar{u}\right)^{2}=\left(\frac{b}{2 l_{d}}\right)^{2}
$$

or $\partial_{z} \bar{u}=1 \pm \sqrt{1-\left(b / 2 l_{d}\right)^{2}}$. Since $\bar{u}$ should vanish as $l_{d} \rightarrow \infty($ or $b \rightarrow 0)$, we have $\partial_{z} \bar{u}=1-\sqrt{1-\left(b / 2 l_{d}\right)^{2}}$. Comparing (4) and (9) while translating from Eulerian to Lagrangian coördinates, we find that Scherk's surface is an array of screw dislocations, i.e., a TGB with:

$$
\begin{aligned}
\tan (\alpha / 2) & =\left(b / 2 l_{d}\right)\left[1-\left(b / 2 l_{d}\right)\right]^{-1 / 2}, \\
\sin (\alpha / 2) & =\pi / l_{d} \\
h(x, y) & =\bar{h}(x \cos (\alpha / 2), y) .
\end{aligned}
$$

Equation (11a) implies that $\sin (\alpha / 2)=b /\left(2 l_{d}\right)$, a standard result from the theory of twistgrain-boundaries, while (11b) shows that $b=2 \pi=2 d$, confirming that there are strength +2 dislocations. Finally, $(\underline{11 d})$ displays the only difference between Scherk's surface and the screw dislocations: Scherk's surface is dilated along the twist axis by $\cos (\alpha / 2)$. We also note that the Eulerian coördinate for Scherk's surface is simply $u(x, y, z)=\bar{u}(x \cos (\alpha / 2), y, z)$.

We now consider the free energies of Scherk's surface and an LSD surface with arbitrary Burgers vector $b=k d$. This free energy has two contributions. The first is the bending energy. In Lagrangian coördinates it can be written as the product of the free energy per surface, the density of surfaces per unit length and the total length of the system. 
We use the Helfrich-Cahn energy for each surface, as has been utilized to study lyotropic monolayers [6]. We find:

$$
F_{\mathrm{b}}=\frac{\kappa L_{z}}{2 d \cos (\alpha / 2)} \int d x d y \sqrt{1+\left(\partial_{x} h\right)^{2}+\left(\partial_{y} h\right)^{2}} H^{2}
$$

where $H$ is the mean curvature of the surface. Note that $\kappa / d=K_{1}$ is the three-dimensional splay modulus of the smectic. Scherk's surface is minimal and thus has $H=0$ - which is precisely why it proves to be a useful ansatz. The LSD surfaces have a non-zero bending energy. Using the standard non-linear expression for $H$ [5], we find

$$
F_{\mathrm{b}}=\frac{\kappa L_{z} \Gamma^{3}}{8 d \cos (\alpha / 2)} \int d \tilde{x} d \tilde{y} \frac{\sinh ^{2}(\tilde{x}) \sin ^{2}(\tilde{y})[\cosh (\tilde{x})-\cos (\tilde{y})]^{-3 / 2}}{[(1+\Gamma) \cosh (\tilde{x})-(1-\Gamma) \cos (\tilde{y})]^{5 / 2}},
$$

where $\Gamma=\tan ^{2}(\alpha / 2)$. Inspection shows that (13) is a convergent integral, proportional to the cross-sectional area of the sample, and that for small angles $F_{\mathrm{b}} \sim b^{4} L_{y} L_{z} /\left(d l_{d}^{5}\right)$. Thus for large $l_{d}$, we see that the interaction energy between two defects scales as $l_{d}^{-5}$. This is different from the usual exponential interactions that a linear theory with both director and displacement fields [7] would predict. Moreover, for fixed angle $\alpha=2 \sin ^{-1}\left(b / 2 l_{d}\right)$, larger values of $b$ are favored over smaller ones. We note that in addition to the mean curvature term in (12), one could also consider a Gaussian curvature term of the form $\bar{\kappa} \int d S K$ where $K$ is the Gaussian curvature. If the elastic constants do not depend on the coördinates $(x, y)$, then the integral of the Gaussian curvature is independent of the surface geometry. The case of varying elastic constants has been considered elsewhere [8].

Since smectic and lamellar layers have a preferred spacing, there is an energy cost for layer compression. The compression energy $F_{\mathrm{c}}$ is most simply written in terms of the Eulerian coördinates $u$ :

$$
F_{\mathrm{c}}=\frac{B}{2} \int d^{3} x\left[\partial_{z} u-\frac{1}{2}(\nabla u)^{2}\right]^{2},
$$

where the nonlinear terms assure complete rotational invariance. The compression energy does not vanish for either LSD surfaces or for Scherk's surface. For Scherk's surface $F_{\mathrm{c}}$ is proportional to the cross-sectional area of the sample in the $y z$-plane:

$$
F_{\mathrm{c}}=\frac{2 B L_{y} L_{z} \sin ^{3}(\alpha / 2)}{\pi \cos (\alpha / 2)} \int_{0}^{\pi / 2} d \theta \int_{0}^{\infty} d \gamma\left[D \cos (2 \theta)-\frac{1}{2} D^{2} \sin ^{2}(\alpha / 2) \sin ^{2}(2 \theta)\right]^{2},
$$

where $D^{-1}=[\cosh (2 \gamma)-\cos (2 \theta)] \sim\left[\gamma^{2}+\theta^{2}\right]$ for $\gamma, \theta \ll 1$. For an LSD surface this compression energy $\bar{F}_{\mathrm{c}}$ is also proportional to the cross-sectional area:

$$
\bar{F}_{\mathrm{c}}=\frac{B L_{y} L_{z} b^{4}}{\pi^{2}\left(2 l_{d}\right)^{3}} \int_{0}^{\pi / 2} d \theta \int_{0}^{\infty} d \gamma D^{2} \cos ^{2}(2 \theta) .
$$


Note that the leading terms in the expansions of $F_{\mathrm{c}}$ and $\bar{F}_{\mathrm{c}}$ in $\alpha$ for $\alpha \ll 1$ are identical when $b=2 d$, i.e., for an LSD surface made of +2 dislocations. Because of the relative sign between the two terms in (15), it is difficult to determine the relative magnitudes of $F_{\mathrm{c}}$ and $\bar{F}_{\mathrm{c}}$ for the same twist angle $\alpha$ and $b=2 d$. However, it is easy to see from (16) that for small angle, strength +1 dislocations lead to a lower compression energy than Scherk's surface.

More importantly, in both cases (LSD and Scherk's surfaces), the integrals diverge for small $\gamma$ and $\theta$. This means that all of them must be cutoff at some short distance $2 \pi / \Lambda \sim d$ as has been observed by Kléman [9]. Inside the cutoff there is a defect core in which the smectic order parameter must vanish. The "core energy" that we find here is actually a nonlinear elastic energy, not to be confused with the usual core energy which arises from the order parameter vanishing at the defect and from relaxation of director modes. We shall use the term "core energy" to refer to the elastic core, keeping in mind that this is only part of the total core energy. The integrals (15) and (16) diverge as $\left(\Lambda l_{d}\right)^{2}$. Thus the dominant contribution to the compression energy for $l_{d} \gg \Lambda^{-1} \sim d$ is

$$
F_{\mathrm{c}} \sim \frac{B b^{4} L_{z}}{l_{d}^{2}} \frac{L_{y}}{l_{d}}\left(\Lambda l_{d}\right)^{2}
$$

This has a simple interpretation: since $L_{y} / l_{d}$ is the total number of dislocations, (17) is the sum over individual dislocations of elastic core energies $\epsilon L_{z}$ with energy per unit length $\epsilon=B b^{4} \Lambda^{2}$. Unlike the bending energy, this energy strongly favors small Burgers vectors,. In addition, we may use (15) or (16) to calculate the leading interaction energy between defects. One finds that the interaction energy is cutoff dependent through logarithmic terms. Therefore, on dimensional grounds the interaction energy per unit length scales as $B b^{4} l_{d}^{-2} \ln \left(\Lambda l_{d}\right)$. The cutoff dependence of the interaction is somewhat unusual: in linear elasticity, defect-defect interactions only depend on the defect separation. Because this system is non-linear, no such decomposition can be easily made.

Since both LSD and Scherk's structures necessarily have core regions, the energy of the core itself must also be taken into account. A detailed analysis of the core structure would be interesting and would allow for explicit energy comparisons between the Scherk and LSD structures. In such a calculation, the core size $2 \pi / \Lambda$ itself would be set so as to minimize the total energy. This is similar to the analysis of defects in lyotropic lamellar systems [10]. Note that there is only one set of parallel core regions, despite the fact that there are two, perpendicular sets of helicoid-like structures in Scherk's surface (1). 
Once the core regions are present in one set of helicoids, the other set no longer consists of perfect helicoids - they are interrupted by the cores of the first set. Thus, one can unambiguously identify a single set of dislocations in the LSD and Scherk structures. This corroborates our earlier claim that Scherk's surface contains a single set of true dislocations, while the second set of (interrupted) helicoids appears only through the geometry of the other defects. Presumably, energy considerations will determine which set of helicoids become dislocations. At $\alpha=\pi / 2$ the energy is degenerate. However, at smaller $\alpha$ the preceding discussion suggests that a system with a larger defect separation $l_{d}$ will have a lower energy. This choice of true topological defects is consistent with the traditional construction of grain boundaries in the TGB-A phase [4]. Finally, we note that Scherk's surface has a non-zero compression energy. The true equilibrium twist grain boundary will adopt a geometry that is an energetic compromise between bending and compression deformations. As a result, it will have a non-vanishing mean curvature, and it will have a structure that is identical to neither Scherk's surface nor to any LSD surface discussed here. It would be interesting to consider a variational ansatz based on Scherk's surface with arbitrary, independent dilations of $x$ and $y$.

We have shown that Scherk's surface is an anisotropic dilation of a periodic surface constructed of a single set of strength 2 screw dislocations. Furthermore since the lamellar ground state has a preferred layer spacing, layer compression contributes to the free energy of the structure. This breaks the dual mapping between the helicoids. It thus follows that Scherk's surface is a twist grain boundary composed of a single set of parallel screw dislocations and that the geometry of these defects creates a perpendicular set of helicoidal structures in the surface. We have also demonstrated that Scherk's surface has a higher energy than an LSD structure built of +1 dislocations for small angles. In the case of biphasic materials, the +1 dislocations we consider here would be topologically forbidden - they would become $+1 / 2$ dislocations. In this case the energetics would be a competition between Scherk's surface and the +2 LSD. In either case, a detailed analysis of the core structure would be required to make an unambiguous prediction of the most stable structure at larger angles, whether it is a distorted Scherk's surface, a distorted LSD surface or some interpolation between them.

We acknowledge stimulating discussions with M. Kléman, B. Pansu and E.L. Thomas. RDK thanks the Université Paris XI, Orsay, where some of this work was done. RDK was supported by NSF CAREER Grant DMR97-32963, an award from Research Corporation and a gift from L.J. Bernstein. TCL was supported by NSF Grant DMR97-30405. 


\section{References}

[1] S.P. Gido, et al., Macromolecules, 26 (1993) 4506.

[2] D.W. Bruce, et al., J. Phys. II France 5 (1995) 289.

[3] A.-M. Levelut, et al., J. Phys. II France, 7 (1997) 981.

[4] S.R. Renn and T.C. Lubensky, Phys. Rev. A 38, 2132 (1988); 41, 4392 (1990).

[5] J.C.C. Nitsche, Lectures on Minimal Surfaces, trans. by J.M. Feinberg, ed. by A. Schmidt (Cambridge University Press, New York, 1989).

[6] Z.-G. Wang and S.A. Safran, J. Phys. (Paris) 51 (1990) 185.

[7] A.R. Day, et al., Phys. Rev. A 27 (1983) 1461.

[8] S.P. Gido and E.L. Thomas, Macromolecules 27 (1994) 849.

[9] M. Kléman, Points, Lines, and Walls: in Liquid Crystals, Magnetic Systems, and Various Ordered Media, (Wiley, New York, 1983).

[10] R.D. Kamien and T.C. Lubensky, J. Phys. II France 7 (1997) 157. 


\section{Figure Captions}

Fig. 1. Twist-grain-boundary structure for +2 screw dislocations. The horizontal lines are the smectic layers at the grain boundary, while the line at $\pm \alpha / 2$ are the layers from the boundary. The very dark set of horizontal lines follows a single smectic layer across grain boundaries. Note that at the grain boundary, the smectic layers are dilated by $1 / \cos (\alpha / 2)$. 


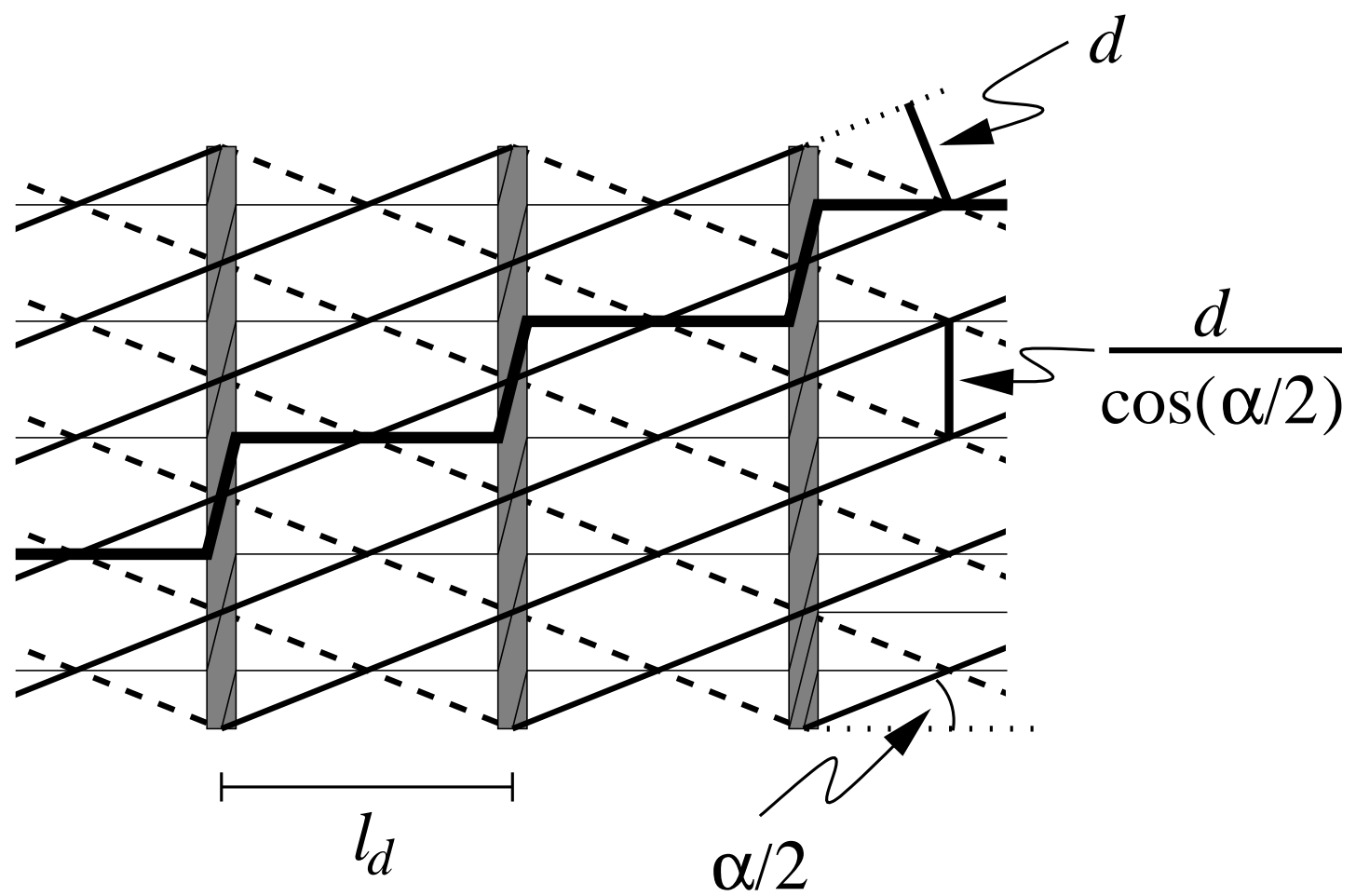

Figure 1 